\title{
Dynamic Properties of Human Brain Structure: Learning-Related Changes in Cortical Areas and Associated Fiber Connections
}

\author{
Marco Taubert, ${ }^{1}$ Bogdan Draganski, ${ }^{1,23 *}$ Alfred Anwander, ${ }^{1 \star}$ Karsten Müller, ${ }^{1}$ Annette Horstmann, ${ }^{1}$ Arno Villringer,,${ }^{1,3}$ \\ and Patrick Ragert ${ }^{1}$ \\ ${ }^{1}$ Max Planck Institute for Human Cognitive and Brain Sciences, Department of Neurology, D-04103 Leipzig, Germany, ${ }^{2}$ Department of Clinical \\ Neurosciences, Centre Hospitalier Universitaire Vaudois, University of Lausanne, 1011 Lausanne, Switzerland, and ${ }^{3}$ Mind Brain Institute, Charité and \\ Humboldt University, D-10117 Berlin, Germany
}

Recent findings in neuroscience suggest that adult brain structure changes in response to environmental alterations and skill learning. Whereas much is known about structural changes after intensive practice for several months, little is known about the effects of single practice sessions on macroscopic brain structure and about progressive (dynamic) morphological alterations relative to improved task proficiency during learning for several weeks. Using T1-weighted and diffusion tensor imaging in humans, we demonstrate significant gray matter volume increases in frontal and parietal brain areas following only two sessions of practice in a complex whole-body balancing task. Gray matter volume increase in the prefrontal cortex correlated positively with subject's performance improvements during a 6 week learning period. Furthermore, we found that microstructural changes of fractional anisotropy in corresponding white matter regions followed the same temporal dynamic in relation to task performance. The results make clear how marginal alterations in our ever changing environment affect adult brain structure and elucidate the interrelated reorganization in cortical areas and associated fiber connections in correlation with improvements in task performance.

\section{Introduction}

The adult brain shows a remarkable capacity for morphological alterations during learning or adaption to a changing environment (Markham and Greenough, 2004; Adkins et al., 2006; Draganski and May, 2008). In human subjects, gray and white matter changes can be observed after intensive long-term motor skill learning for several months (Draganski et al., 2004; Boyke et al., 2008; Scholz et al., 2009). Even though we face a consistently changing environment in our daily life and the need to rapidly adapt to such changes, less is known about the capability of the adult human brain for structural alterations in response to slight environmental changes. Animal studies suggest that formation of new synaptic connections by dendritic spine growth and remodeling of axons is associated with experience-dependent behavioral changes (Trachtenberg et al., 2002; Chklovskii et al., 2004; Markham and Greenough, 2004; DeBello, 2008; Butz et al., 2009; $\mathrm{Xu}$ et al., 2009). For example, motor skill learning rapidly forms and eliminates dendritic spines in response to short practice sessions (Xu et al., 2009).

Behavioral studies of motor skill learning indicate that individuals pass through different learning phases during the time

\footnotetext{
Received May 20, 2010; revised July 2, 2010; accepted July 9, 2010.

*B.D. and A.A. contributed equally to this work.

Correspondence should be addressed to Dr. Patrick Ragert, Max Planck Institute for Human Cognitive and Brain Sciences, Department of Neurology, Stephanstrasse 1a, D-04103 Leipzig Germany. E-mail: ragert@ cbs.mpg.de. DOI:10.1523/JNEUROSCI.2567-10.2010

Copyright $\odot 2010$ the authors $\quad 0270-6474 / 10 / 3011670-08 \$ 15.00 / 0$
}

course of skill acquisition (Lee and Swinnen, 1993; Newell, 1996; Karni et al., 1998). Functional neuroimaging studies show recruitment of specific brain networks during early and late phases of skill learning demonstrating distinct dynamic patterns of neural activity (Karni et al., 1995; Floyer-Lea and Matthews, 2005; Luft and Buitrago, 2005). So far, however, the structural implementation of such behavioral and functional adaptations within distinct brain areas and their associated structural connectivity patterns across the phases of learning is largely unexplored. Moreover, it remains elusive whether the temporal dynamics of such changes in the human brain are directly linked to improvements in motor performance over time. Motor skill learning and the organization of goal-directed behavior have been associated with neural activity changes in premotor, parietal, and prefrontal cortex as well as in their functional connectivity patterns (Passingham, 1993; Andres et al., 1999; Koechlin et al., 1999; Swinnen and Wenderoth, 2004; Koechlin and Hyafil, 2007; Sun et al., 2007; Nachev et al., 2008; Boorman et al., 2009).

In the present study, we hypothesized that (1) a short period of practice in a complex motor task induces significant changes in brain structure and (2) long-term motor skill learning is associated with dynamic patterns of structural alterations in functionally relevant brain areas and corresponding anatomical projections in correlation with improvements in task performance.

We used voxel-based analysis of T1- and diffusion-weighted image (DWI) magnetic resonance (MR) data (Ashburner and Friston, 2000) in young healthy volunteers in a multilevel longitudinal 
design during 6 weeks of learning a complex whole-body balancing task. We introduced a novel parameter data processing approach to adjust for volume change-related biases of DWI data analysis. We anticipated that the complementary character of voxel-based analysis of distinct DWI parameters would allow more straightforward interpretation of the hypothesized learning-induced structural changes.

\section{Materials and Methods}

Subjects. Twenty-eight healthy, right-handed (Oldfield, 1971) subjects (mean age, 25.9 years; SD, 2.8 years; 14 females) with normal or corrected-to-normal vision were recruited for this study after obtaining written informed consent approved by the local ethics committee. All subjects underwent a neurological examination before participation. Subjects were naive to the experimental setup with no prior experience of other highly coordinative balancing skills.

Experimental overview. Fourteen subjects were asked to learn a wholebody dynamic balancing task (DBT) over six consecutive weeks with one training day (TD) in each week (see Fig. $1 A$ ). TDs as well as the time schedule in each week was kept constant $( \pm 1 \mathrm{~d})$ across the whole learning period. On each TD, subjects performed the DBT for $\sim 45 \mathrm{~min}$. During task performance, electromyographical (EMG) activity of the left and right soleus muscle was recorded continuously to capture possible changes in muscle activity pattern. MR data acquisition was performed as follows: baseline scan (s) before learning ( $\mathrm{s} 1$, pre), two intermittent scans after 2 and 4 weeks (s2 and s3), and a final scan 1 week after completion of the learning period (s4) (see Fig. $1 \mathrm{~A}$ ). Importantly, MR scanning was performed prior to the practice session on TD1, TD3, and TD5 (see Fig. $1 A$ ). The control group, consisting of 14 age- and gender-matched subjects scanned at baseline (s1, pre) and 2 weeks later (s2), did not practiced the balancing task.

Whole-body dynamic balancing task. The DBT was performed on a movable platform with a maximum deviation of $26^{\circ}$ to each side (stability platform, model $16030 \mathrm{~L}$, Lafayette Instrument). Subjects were instructed to stand with both feet on the platform and to keep it in a horizontal position as long as possible during a trial length of $30 \mathrm{~s}$.

To familiarize subjects with the task and to prevent falls in the initial three trials on TD1, we allowed use of a supporting hand rail. The familiarization trials were excluded from the analysis. The behavioral outcome measure was the time (in seconds) in which subjects kept the platform in a horizontal position (designated BAL) within a deviation range of $\pm 3^{\circ}$ to each side out of the total trial length of $30 \mathrm{~s}$. We used a discovery learning approach (Wulf et al., 2003; Orrell et al., 2006) in which no information about the performance strategy was provided during learning. After each trial, subjects were only given verbal feedback about their time in balance (BAL). Therefore, subjects had to discover their optimal strategy to improve task performance (e. g. error correction strategy with legs, hip, and arms) based on trial outcome by trial and error. On each of the six TDs, 15 trials had to be performed with an intertrial interval of 2 min to avoid fatigue. Thus, the time to complete the DBT on each TD was $\sim 45 \mathrm{~min}$. Three months after the end of the learning period, the stability of the acquired motor skill was reassessed in a retention test in 13 subjects.

Surface EMG recordings. Ag-AgCl surface electrodes were positioned bilaterally on the skin overlying the soleus muscle (SM) of the right and left leg in a bipolar montage (interelectrode distance, $\sim 5 \mathrm{~cm}$ ). Electrode positions were carefully determined and kept constant to ensure identical recording sites during the learning period. The signal was amplified using a Counterpoint EMG device (Digitimer D360) with bandpass filtering between 50 and $2000 \mathrm{~Hz}$, digitized at a frequency of $5000 \mathrm{~Hz}$, and fed off-line to a data acquisition system for further analysis (CED 1401 system, Spike2 software, Cambridge Electronic Devices). EMG activity was recorded continuously during DBT and subjects were instructed to relax as much as possible during the rest periods of the task. EMG signals were processed offline with a low pass filter. Rectified EMG activities for right and left SM were calculated as a mean average voltage starting from the EMG onset of each trial. Then, muscular imbalances were calculated as the ratio between left and right SM EMG activity for each trial (where a value of 1 indicates no EMG difference and values $>1$ or $<1$ higher EMG activity on the left or right SM).

Statistical analysis of behavioral and EMG data. Repeated measures ANOVA with (1) factor TIME (TD1, TD2, TD3, TD4, TD5, and TD6) and (2) factor TRIAL (trial 1 , trial $2, \ldots$, trial 15) for each TD were performed to identify significant improvements in motor performance (1) across the whole training period and (2) within each TD. Then, paired $t$ tests (two-tailed) were conducted to identify improvements between consecutive TDs. Therefore, we averaged time in balance (BAL) for each subject on each TD ( 15 trials). Consolidation between subsequent TDs was assessed by comparing the last trial of the previous TD and the first trial of the subsequent TD (two-tailed paired $t$ test). Retention of motor performance was expressed in percentage relative to average performance on the last training day (TD6) for each subject (e.g., 100\% retention indicates maximal stability of acquired motor skill). Furthermore, we identified regaining efforts by comparing performance levels on the last trial on TD6 with initial retention trials in each subject (two-tailed paired $t$ test) (Ryan, 1965). We applied Bonferroni's correction for multiple comparisons at the threshold of $p<0.05$. EMG recordings were analyzed using repeated measures ANOVA with factor TIME (TD1, TD2, TD3, TD4, TD5, and TD6). Additionally, we tested for correlation between muscular imbalances and individual performance improvement during training. In general, repeated measures ANOVAs were performed, if necessary, with a Greenhouse-Geisser sphericity correction.

Image acquisition. MR imaging (MRI) data was acquired on a 3T Magnetom Tim Trio scanner (Siemens) using a 32 channel head coil. We used the same protocol for each volunteer and each scanning session. In each scanning session, we acquired whole brain diffusion weighted images with a double spin echo sequence $\left[60\right.$ directions; $b$-value $=1000 \mathrm{~s} / \mathrm{mm}^{2}$; 88 slices; voxel size, $1.7 \times 1.7 \times 1.7 \mathrm{~mm}$, no gap; repetition time $(\mathrm{TR})=$ $12.9 \mathrm{~s}$; echo time $(\mathrm{TE})=100 \mathrm{~ms}$; field of view $($ FOV $)=220 \times 220 \mathrm{~mm}$; parallel acquisition GRAPPA (generalized autocalibrating partially parallel acquisition) acceleration factor 2] plus seven volumes without diffusion weighting $\left(b=0 \mathrm{~s} / \mathrm{mm}^{2}\right)$ at the beginning of the sequence and after each block of 10 diffusion weighted images as anatomical reference for offline motion correction. T1-weighted images were acquired using a MPRAGE (magnetization-prepared rapid acquisition gradient echo) sequence $\left(\mathrm{TR}=1.3 \mathrm{~s} ; \mathrm{TE}=3.46 \mathrm{~ms}\right.$; flip angle $=10^{\circ}, \mathrm{FOV}=256 \times 240$ $\mathrm{mm}$; 176 sagittal slices; voxel size $=1 \times 1 \times 1.5 \mathrm{~mm}$ ). The acquisition time for the anatomical MRI and diffusion-weighted scan was $13 \mathrm{~min}$ and 15 min respectively.

MRI data processing and analysis. Pre-processing of T1-weighted images was performed using SPM5 (Wellcome Trust Centre for Neuroimaging, University College London, London, UK; http://www.fil.ion.ucl. ac.uk/spm), implemented in VBM (voxel-based morphometry) Toolbox 5.1 (http://dbm.neuro.uni-jena.de/vbm.html) running under a Matlab environment (Mathworks, version 7.7). We applied standard VBM 5.1 routines and default parameters. Images for each scanning time point were bias corrected, segmented, and registered (using rigid-body transformation with translation and rotation about the three axes) to standardized Montreal Neurological Institute (MNI) space using the "unified segmentation" approach (Ashburner and Friston, 2005). We processed separately the data acquired at each time point. Gray matter (GM) segments were scaled by the Jacobian determinants of the deformations to account for local compression and expansion during linear and nonlinear transformation (i.e., "modulation"). Finally, the modulated GM volumes were smoothed with a Gaussian kernel of $8 \mathrm{~mm}$ full width at half maximum (FWHM).

First, we tested for increases and decreases in gray matter from baseline (s1) to the learning period (s2, s3, s4) using TIME (s1, s2, s3, s4) as factor in a full-factorial design. Second, we were interested in the temporal dynamics of GM changes across the four scanning time points relative to motor performance improvements and changes in muscular imbalances. Therefore we used a whole-brain parametric correlation analysis embedded in a full factorial design with factor SUBJECT (1-14), with each level containing the four scans (s1, s2, s3, s4) for each subject. Specifically, we looked for brain regions that show a direct linear relationship to performance improvements for each subject across the whole brain, consisting of mean performance of the initial five trials on TD1 and mean perfor- 
mance for TD2, TD4, and TD6. Furthermore, we looked for regions across the whole brain that show a direct linear relationship to individual improvements in muscular imbalances using zero as a baseline value and individual imbalance adaptations for each subject to TD2, TD4, and TD6. Additionally, GM changes between distinct scanning time points were evaluated using paired $t$ test.

For statistical analysis, we excluded all voxels with a GM value below 0.2 (with a maximum value of 1 ) to avoid possible partial volume effects near the border between GM and white matter (WM). For each analysis, cluster size was corrected according to the local smoothness values using nonstationary cluster extent correction at $p<0.05$ (Hayasaka et al., 2004). We report effects for clusters of voxels exceeding a voxel level threshold of $p<0.001$ (uncorrected) and a cluster size threshold at $p<$ 0.05 , family-wise error (FWE) corrected for multiple comparisons in the context of Gaussian random field theory (Friston et al., 1996).

Preprocessing of diffusion-weighted images and analysis. We included fractional anisotropy (FA) to investigate directionally dependent changes in water diffusion. Furthermore, axial diffusivity $\left(\lambda_{\|}\right)$and radial diffusivity $\left(\lambda_{\perp}\right)$ provided further information about the source of FA changes, since FA is based on the relation between $\lambda_{\|}$and $\lambda_{\perp}$. In addition, we analyzed mean diffusivity (MD) to find directionally independent changes in the amount of water diffusion.

The initial step of diffusion MR image processing was motion correction using images without diffusion weighting (i.e., $b 0$ images) and rigid body transformations. In the same process, the diffusion MR images were spatially coregistered to the individual T1-weighted image using rigid body transformation and interpolated to $1 \mathrm{~mm}^{3}$ voxel size. Subsequently, we computed for each voxel a diffusion tensor (Mori and Zhang, 2006) and characteristic diffusion tensor imaging (DTI) contrast parameter (FA, MD, $\lambda_{\|}$, and $\lambda_{\perp}$ ). Affine registration was performed intraindividually between FA maps and white matter segments (from T1weighted images). Maps of parallel, perpendicular, and mean diffusivity (intrinsically in the same native space as FA maps) were subsequently coregistered to the white matter segments using the parameters estimated in the previous step. $\mathrm{FA} / \mathrm{MD} / \lambda_{\|} / \lambda_{\perp}$ maps were linearly and nonlinearly normalized using the deformation fields estimated in the registration step of the subject's specific T1-weighted image (from VBM procedure described above). Additionally, to adjust $\mathrm{FA} / \mathrm{MD} / \lambda_{\|} / \lambda_{\perp}$ data for linear and nonlinear effects of registration, we performed scaling procedure allowing us to eliminate bias from white matter volume changes (Lee et al., 2009) as shown in the equation:

$$
\text { scaled_DTI }=\frac{s\left(\mathrm{wDTI}{ }^{\star} \mathrm{mwWM}\right)}{\mathrm{smwWM}},
$$

where $s$ is smoothed data, $w$ is warping, $m$ is modulation, $W M$ is individual white matter segment from T1-weighted image, and DTI is individual $\mathrm{FA}, \mathrm{MD}, \lambda_{\|}$, or $\lambda_{\perp}$ image. Smoothing was performed with a spatial Gaussian kernel of $8 \mathrm{~mm}$ FWHM.

Similar to the GM analysis, we tested for FA/MD changes from baseline ( $\mathrm{s} 1)$ to the subsequent learning phase (s2, s3, s4) using TIME (s1, s2, s3, s4) as factor in a full-factorial design. We then used whole-brain parametric correlation analysis with factor SUBJECT (1-14) to look for white matter regions that show a direct linear relationship to motor performance improvements and adaptations in muscular imbalances across the four scanning time points (see above). Additionally, we tested for a direct linear relationship between performance improvements and changes in parallel $\left(\lambda_{\|}\right)$and perpendicular diffusivity $\left(\lambda_{\perp}\right)$ in prefrontal regions where we observed performance-related GM and FA changes (see Fig. $4 A, B$ ) using a region of interest (ROI) approach (small-volume correction with a sphere diameter of $20 \mathrm{~mm}$ centered at peak voxel from the GM parametric correlation analysis).

We excluded all voxels with FA values below 0.2 to isolate white matter from the rest of the brain. Cluster size was corrected according to the local smoothness values using nonstationary cluster extent correction (Hayasaka et al., 2004). Effects were reported for clusters of voxels exceeding a cluster size threshold of $p<0.05$, FWE corrected for multiple comparisons in the context of Gaussian random field theory and a voxel level threshold of $p<0.001$ (uncorrected). For ROI analysis, we used FWE correction at $p<0.05$.
Identification of primary and secondary fiber directions. Using a crossing fiber model, we computed in each voxel two scalars, $\mathrm{fl}$ and $\mathrm{f} 2$, indicating the contribution of each fiber (f) compartment to the measured signal (see below). Following Jbabdi et al. (2010) these scalar values must be reassigned to obtain a consistent labeling across subjects that is not assured by the model used. Compared to Jbabdi et al. (2010) we used a slightly different procedure to compute this reassignment using the smooth and robust primary fiber direction provided by the simple single fiber diffusion tensor. In each voxel we compared the direction of both crossing fiber orientations with the main fiber orientation using a single tensor model. The direction with the smaller inclosing angle was identified as primary direction. The scalar values $\mathrm{f} 1$ and $\mathrm{f} 2$ were reassigned accordingly. This procedure resulted in a robust identification of fiber compartments across subjects (Fig. S10, available at www.jneurosci.org as supplemental material).

Calculating mean distribution of crossing fibers. Distribution of crossing fibers was estimated using FMRIB's Diffusion Toolbox implemented in FSL (Behrens et al., 2007; Jbabdi et al., 2010) [Analysis Group, Functional MRI of the Brain (FMRIB), Oxford, UK; http://www.fmrib.ox.ac.uk/fsl/ fdt/index.html). The software (bedpostx) allowed us to model and automatically determine the distribution of crossing fibers in each voxel of the brain. Specifically, $f$ values for the probability of major (f1) and secondary (f2) fiber direction were calculated for all acquired diffusion MRIs. Then, mean $\mathrm{f} 1$ and $\mathrm{f} 2$ values were calculated for three ROIs in left ventral and dorsal prefrontal and right parietal white matter, corresponding to the significant clusters from the whole-brain parametric FA analysis (Fig. $4 B$; Fig. S9, available at www.jneurosci.org as supplemental material). Voxel with $f$ values $<0.05$ (no significant contribution of the first or second fiber compartment) were discarded in the $\mathrm{fl}$ or $\mathrm{f} 2$ maps, respectively.

\section{Results}

\section{Behavioral and electrophysiological results}

Performing the whole-body DBT on six consecutive weeks with one TD each week resulted in significant performance improvements (Fig. 1C). Time in balance increased from $6.2 \pm 0.1 \mathrm{~s}$ (mean \pm SEM) in the initial trial of TD1 to $20.1 \pm$ $0.2 \mathrm{~s}$ in the last trial of TD6 (repeated measures ANOVA; main effect of TD: $F_{(5,78)}=45.8 ; p<0.001$, Greenhouse-Geisser corrected; see also Fig. S1, available at www.jneurosci.org as supplemental material).

Furthermore, we found a significant decrease in muscular imbalance between both muscles during the time course of learning (repeated measures ANOVA; main effect of TD: $F_{(5,65)}=17.072$; $p<0.001$, Greenhouse-Geisser correction) (Fig. $1 D$ ) indicating higher EMG activity on the left (nondominant) as compared to the right (dominant) SM [left-right ratio $1.23 \pm 0.04$ (mean \pm SEM)] at the beginning of the learning period (TD1). However, on TD6 this side-to-side difference was significantly reduced, representing a diminished muscular imbalance $(0.95 \pm 0.03)$. We also found a negative linear correlation $(r=-0.53, p<0.0001$. across subjects and TD) between muscular imbalances and motor performance (see Fig. 1D).

\section{Imaging results}

Structural changes during learning ( 1 to s2, s3, s4)

We demonstrate significant GM volume increase in left supplementary motor areas (SMA), left superior frontal gyrus (SFG), and left medial orbitofrontal cortex (OFC; orbital part of the medial frontal gyrus) (Fig. 2; Table S1, available at www. jneurosci.org as supplemental material) during 6 weeks of DBT learning. We additionally report GM decrease in right putamen, right inferior orbitofrontal cortex (iOFC; orbital part of the inferior frontal gyrus), left inferior occipital gyrus, right middle temporal gyrus (MTG), and lobule VIII in cerebellum bilaterally (Table S5, Fig. S5, available at www.jneurosci.org as supplemen- 
A

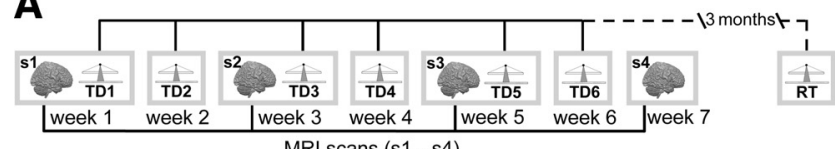

B
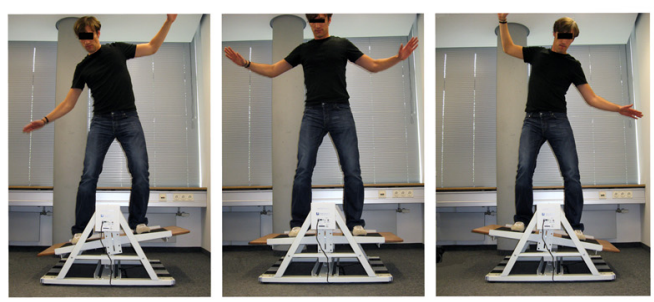

C

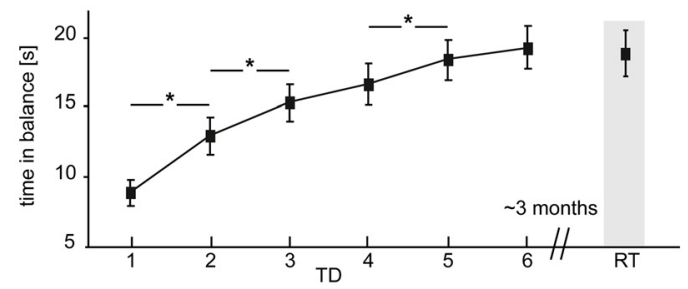

D

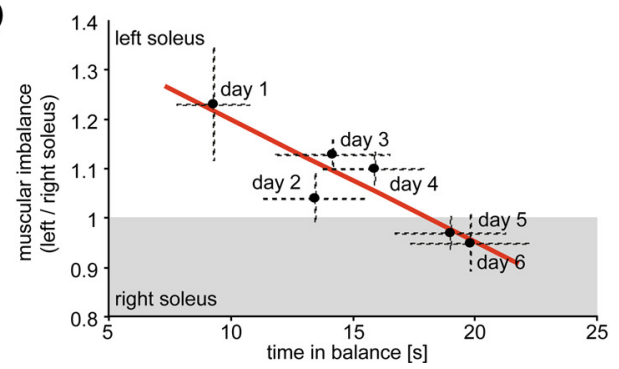

Figure 1. Experimental design and behavioral and electrophysiological results. $\boldsymbol{A}$, Experimental design. Subjects performed 15 trials ( $\sim 45 \mathrm{~min}$ ) of the whole-body DBT on each of the six TDs as well as in the retention test (RT) and received verbal feedback about their performance after each trial (see below). Before the learning session on TD1, TD3, TD5, and in the seventh week, structural MRI scans were performed to assess learning-related gray and white matter changes. $\boldsymbol{B}$, DBT. Subjects were instructed to keep a balance platform in a horizontal position as long as possible during a trial length of $30 \mathrm{~s}$. Motor performance was determined as the time $\{\mathrm{s}$ ) in which the subjects kept the platform in a horizontal position, within a deviation range of \pm $3^{\circ}$ to each side, out of the total trial length of $30 \mathrm{~s}$ (BAL). C, Behavioral results. Improvements in motor performance during the time course of learning as well as mean retention performance of 95\% (in percentage of mean performance on TD6) after 3 months without training (filled squares, mean performance across subjects; error bars, SEM). Asterisks indicate significant improvements in motor performance between consecutive training days (TD1 to TD2, $p<0.001$, TD2 to TD3, $p<0.001$; TD4 to TD5, $p<0.001$; see also Fig. S1, available at www.jneurosci.org as supplemental material, for within-session improvements). $\boldsymbol{D}$, Negative correlation between muscular imbalances and motor performance during the learning period (dotted lines indicate SD for muscular imbalances and motor performance; see also Fig. S2, available at www. jneurosci.org as supplemental material).

tal material). We identified significant FA decrease in bilateral prefrontal WM regions (Fig. 2, Table S2, available at www. jneurosci.org as supplemental material). Topographically, the FA findings are in close spatial proximity to GM changes in left SFG and right MFG (Fig. 2). Furthermore, we found an MD increase in right inferior parietal and right cerebellar WM regions (Table S3, available at www.jneurosci.org as supplemental material). No FA increase or MD decrease were detected in these analyses.

Rapid structural changes during learning ( 1 to s2)

VBM analyses on data corresponding to the first 2 weeks of learning (after $2 \times 45$ min of practice) showed significant GM expansion in bilateral SMA, left SFG, right MFG, and left supramarginal gyrus (SMG) (Fig. 3A, Table S1, available at www.jneurosci.org as sup- plemental material). We were not able to detect any GM reduction during this period $(s 1>s 2)$. The initial GM changes could no longer be detected on s3. FA significantly decreased in WM regions adjacent to the left lateral prefrontal cortex and right M1 (Table S2, available at www.jneurosci.org as supplemental material). MD increased in right inferior parietal WM regions (Table S3, available at www.jneurosci.org as supplemental material). We did not detect any significant changes in GM and FA in the ageand gender-matched control group ( $1<\mathrm{s} 2 ; n=14)$. Significant GM and FA changes in the learning group were confirmed by interaction analyses with the control group (Figs. S7, S8, available at www.jneurosci.org as supplemental material).

Parametric correlation with improvements in motor performance The multiple regression analysis between GM volume and motor performance parameter across all time points (s1, s2, s3, s4) showed positive correlation in the left superior orbitofrontal cortex (sOFC; orbital part of the superior frontal gyrus) (Fig. $4 \mathrm{~A}$, Table S1, available at www.jneurosci.org as supplemental material). In contrast, GM changes in right iOFC, right SMG, left inferior occipital gyrus, right MTG, and left cerebellum correlated negatively with improvements in motor performance (Table S5, Fig. S5, available at www.jneurosci.org as supplemental material).

Additionally, we detected negative correlation between performance improvement and FA in the left prefrontal and in the right inferior parietal WM (Fig. 4B, Table S2, available at www. jneurosci.org as supplemental material). All significant regions in the FA correlation analysis (Fig. $4 B$ ) showed high probability of crossing fibers within these regions (Fig. S9, available at www. jneurosci.org as supplemental material).

MD correlated negatively with motor performance parameters in bilateral anterior centrum semiovale, right internal capsule, and left brainstem (Fig. 4C, Table S3, available at www. jneurosci.org as supplemental material). Positive linear correlation between performance improvement and MD was detected in right inferior parietal and right superior temporal WM (Table S3, available at www.jneurosci.org as supplemental material).

In a post hoc correlation analysis with motor performance parameter restricted to the left sOFC, we estimated parallel diffusivity $\left(\lambda_{\|}\right)$and perpendicular diffusivity $\left(\lambda_{\perp}\right)$. Both parallel and perpendicular diffusivity showed a significant negative correlation with performance improvements (Table S4, available at www.jneurosci.org as supplemental material).

Parametric correlation with changes in muscular imbalances GM in the left SMA was positively correlated with individual adaptations in muscular imbalances during the whole learning period (Fig. 3B, Fig. S3, Table S1, available at www.jneurosci.org as supplemental material). Negative correlation between FA and muscular imbalances were detected in left superior parietal and right occipital WM and left cingulum (Table S2, available at www.jneurosci.org as supplemental material). Also in left superior parietal WM, MD correlated negatively with muscular imbalances. We found positive correlations between $\mathrm{MD}$ and muscular imbalances in cerebellum bilaterally (Table S3, available at www.jneurosci.org as supplemental material).

\section{Discussion}

In this longitudinal study, we demonstrate substantial GM volume expansion in frontal and parietal brain areas after only two practice sessions in a complex whole-body balancing task. The distinct temporal dynamics of structural GM changes confirm the functional significance of distinct brain regions during differ- 
ent phases of motor skill acquisition (Fig. $5 A$ ). Additional confirmation about the behavioral relevance of structural changes comes from the positive correlation between GM volume and performance improvements over time. Changes in WM tissue properties paralleled the spatial and temporal pattern of GM volume changes to indicate the major impact of motor skill learning on cortical structures and their associated long-range axonal connections (Fig. 5B). Our findings reveal the enormous potential of the adult human brain to undergo learning-induced structural adaptations in response to a few minutes of practice and elucidate possible underlying mechanisms of brain plasticity.

\section{Rapid and transient structural gray matter changes}

Our results demonstrate that only 90 min of practice in a complex balancing task, distributed over 2 weeks, resulted in macroscopic structural GM alterations. We did not detect any GM changes in a control group not exposed to the task, minimizing the potential impact of external factors on brain structure. We conclude from this that longitudinal VBM analysis is sensitive to GM changes following short periods of motor practice, which could be used as independent biomarker monitoring the efficacy of neurorehabilitation training schedules (Langhorne et al., 2009) or occupational skill acquisition (Moulton et al., 2006). We also found that initial GM changes in sensorimotorrelated regions decreased in the later learning phase, while GM in prefrontal cortex continuously increased. We interpret this finding as the initial challenge of learning a complex motor skill and an important characteristic for entering later learning phases (Lee and Swinnen, 1993; Newell, 1996; Schmidt and Lee, 1999; Driemeyer et al., 2008). Specifically, we found transient GM changes in premotor and inferior parietal areas, which are known to be associated with complex motor skill acquisition and integration of vestibular signals for postural control (Guldin and Grusser, 1998; Swinnen and Wenderoth, 2004; Dieterich and Brandt, 2008; Nachev et al., 2008). This supports the notion of complex multijoint movement acquisition (error correction strategies, e.g., with hip and arms) in response to vestibular input during wholebody perturbations. In this regard, inferior parietal changes may result from an increased accuracy and speed of detecting and specifying whole-body perturbations for dynamic balance control.

\section{Biological substrates of gray matter changes}

The exact nature of the underlying cellular mechanisms of learning-induced GM changes in humans still remains elusive A, anterior.

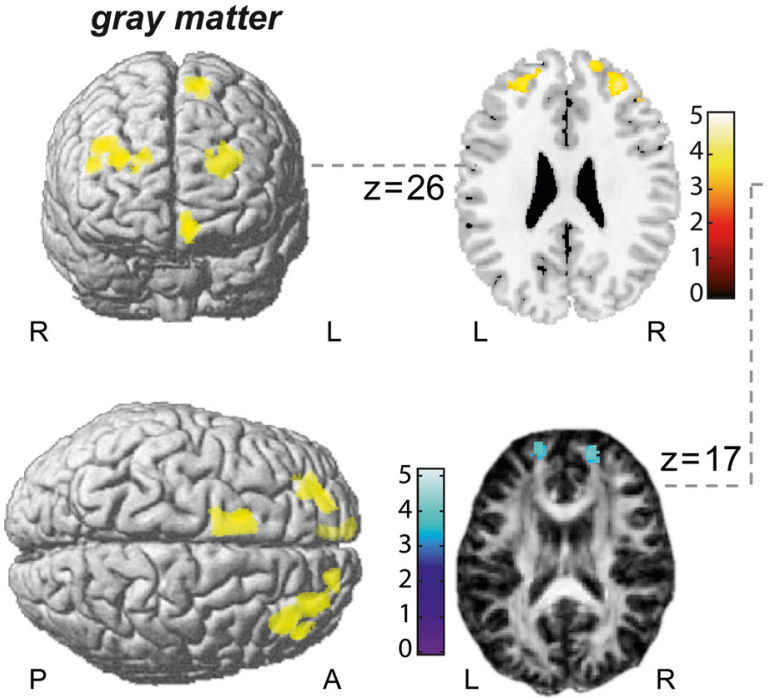

white matter FA

Figure 2. Increase in GM volume and decrease in WM FA across the whole learning period (s1-s2, s3, s4). These analyses are independent from improvements in motor performance. GM (yellow) and WM (cyan) changes in bilateral lateral prefrontal regions are shown in axial sections overlaid on MNI colin27 image (top image) and individual (normalized) FA map (bottom image). Images are shown at $p<0.05$ (corrected). Bars indicate $t$ values. R, Right; L, left; $P$, posterior; A, anterior.
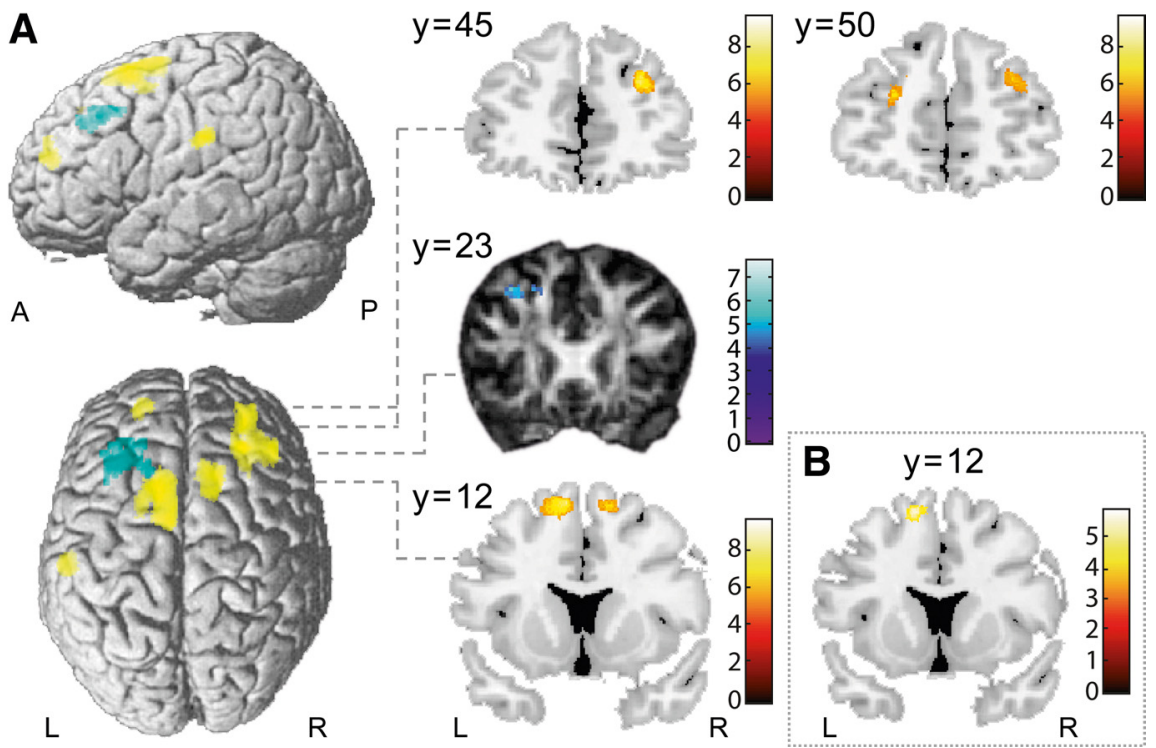

Figure 3. $A$, Rapid changes in GM volume and WM FA from $s 1-s 2$ (paired $t$ test). Rendered brains indicate GM expansion (yellow) and FA decrease (cyan). Top image shows left side of the brain. Upper two coronal sections show GM expansion in bilateral lateral prefrontal cortex. Middle coronal section shows FA decrease in left prefrontal WM. Bottom left section indicates GM expansion in bilateral supplementary motor areas. $\boldsymbol{B}$, Positive linear correlation between GM expansion in left supplementary motor areas and individual adaptations in muscular imbalances across the whole learning period (Fig. S3, available at www. jneurosci.org as supplemental material). GM and WM changes are shown in coronal sections overlaid on MNI colin27 image and individual (normalized) FA map. All images are shown at $p<0.05$ (corrected). Bars indicate $t$ values. R, Right; L, left; P, posterior;

(May and Gaser, 2006; Draganski and May, 2008). Our data showed a rapid GM increase in sensorimotor-related brain areas as early as after two training days $(2 \times 45 \mathrm{~min})$ that were separated by 1 week which, subsequently decreased to the last 4 weeks. A recent study in humans also found macroscopic changes in GM as early as after 5 days of continuous intervention with repetitive transcranial magnetic stimulation (May et al., 2007). Together, these results support the idea of rapid intracortical remodeling of dendritic spines and axonal terminals as a possible biological substrate for GM changes in humans (Trachtenberg et al., 2002; 
A

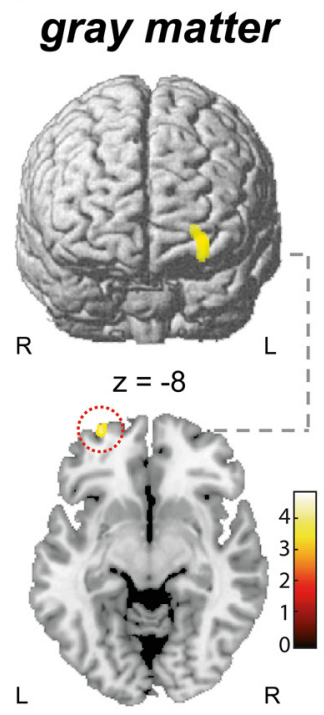

B

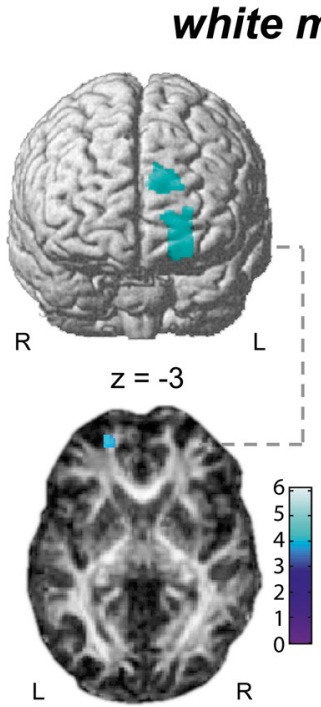

C white matter MD

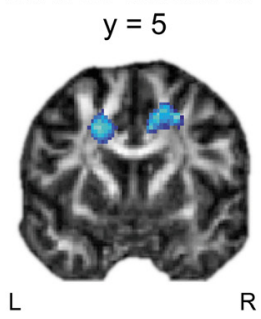

$\mathrm{P}$

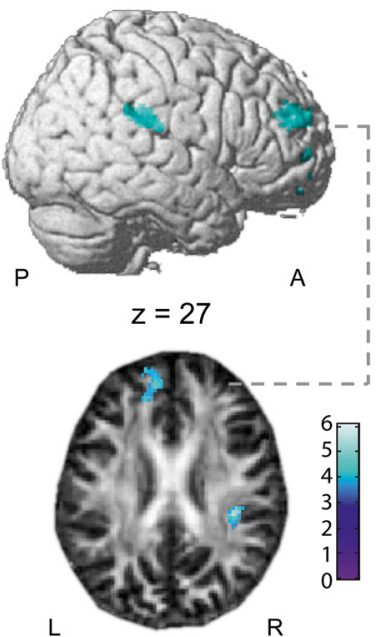

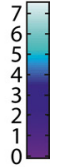

$\mathrm{R}$

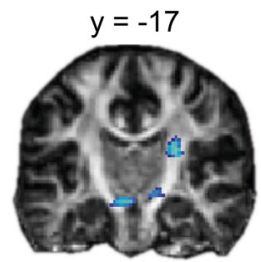

$\mathrm{R}$

Figure 4. Parametric correlation between individual improvements in motor performance across the whole learning period ( $s 1, s 2, s 3$, and s4) and GM volume and WM, FA and MD. $A$, Positive linear correlation between improvements in motor performance and GM expansion in left SOFC. GM changes are shown in axial section (red dotted circle). $\boldsymbol{B}$, Negative linear correlation between improvements in motor performance and FA changes in left prefrontal and right parietal WM regions (cyan). Right rendered brain represents right hemisphere. FA changes are shown in axial sections. C, Negative linear correlation between improvements in motor performance and MD changes in bilateral anterior centrum semiovale, left brainstem and right internal capsule (cyan). All images are shown at $p<0.05$ (corrected). Bars indicate $t$ values. $R$, Right; L, left; $P$, posterior; $A$, anterior.

A

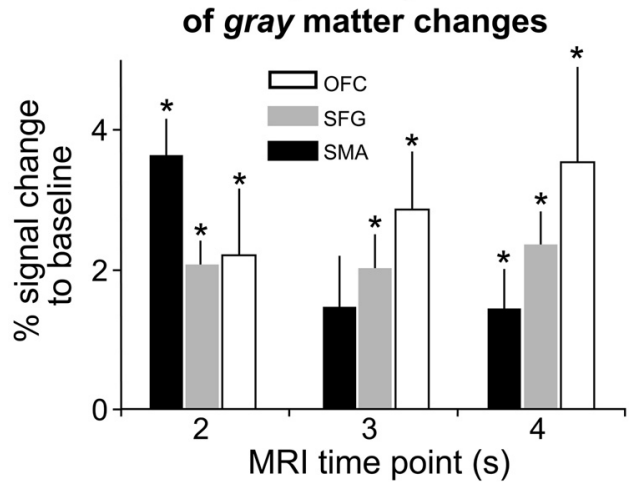

B

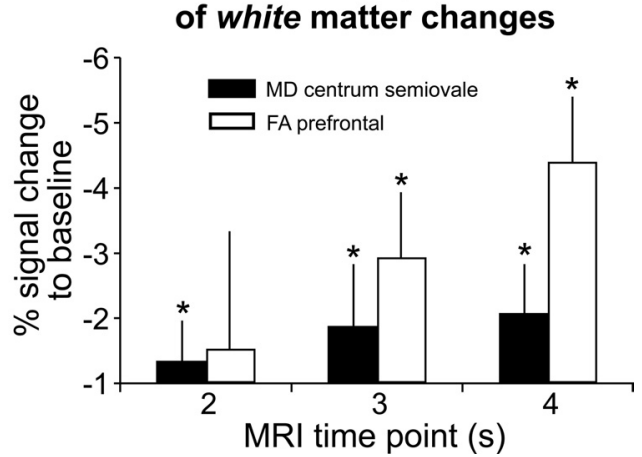

Figure 5. Temporal dynamics of GM and WM FA and MD changes during learning. $A, G M$. Diagram shows percentage signal change (error bars indicate SEM) on $s 2$, s3, and $s 4$ relative to baseline (S1) in peak voxel in left OFC, SFG, and SMA. B, WM. Diagram shows percentage signal change (error bars indicate SEM) in FA/MD during learning in peak voxel in WM regions adjoining left $\mathrm{OFC}$ and in left anterior centrum semiovale. Asterisks indicate significant changes in peak voxel intensity compared to baseline (s1) intensity at $p<0.05$ (paired $t$ test).
Chklovskii et al., 2004; Markham and Greenough, 2004; DeBello, 2008; Butz et al., 2009; Xu et al., 2009). Furthermore, glia cells have been shown to support synaptic efficacy, and its hypertrophy is associated with an increase in synapse formation during motor skill learning (Anderson et al., 1994), representing a further candidate for the observed macroscopic GM changes in humans.

Slowly evolving GM changes and the capability for skilled movement

In the present study, we demonstrate an association between GM changes in the prefrontal cortex and parameters of motor skill acquisition in humans. We suggest that GM changes in sOFC reflect a build-up and maintenance of an optimal task-specific strategy as a result of exploring and evaluating potential movement coordination patterns for task execution based on reinforcement learning supported by ventromedial prefrontal cortex (Fig. S4, available at www.jneurosci.org as supplemental material) (Koechlin and Hyafil, 2007; Kable and Glimcher, 2009). The anterior prefrontal cortex is responsible for the exploration of alternative behavioral options during execution of a prevailing behavioral plan (Koechlin and Hyafil, 2007). Importantly, this feature is crucial for the development of an optimal strategy for task performance during complex motor skill learning (Newell et al., 1989; Lee and Swinnen, 1993; Newell, 1996; Caillou et al., 2002). In our study, we used a discovery learning approach (Wulf et al., 2003; Orrell et al., 2006), which means that at no time during learning did we provide subjects with instructions about how to best perform the task, thus giving them the opportunity to find their own optimal strategy to perform the DBT. Subsequent interviews and visual inspection of video taped task execution confirmed that subjects explored and evaluated different movement coordination patterns (error correction strategies. e.g., with hip and arms) to improve performance in the DBT. Furthermore, prediction of final performance level (on TD6) by prelearning gray matter volume in the temporal cortex (Fig. S6, available at www.jneurosci.org as supplemental material) suggests an impact 
of long-term memory representations on evolving task-related performance strategies (Zanone and Kelso, 1992).

\section{Microstructural changes in WM and biological substrates}

It is noteworthy that the areas of negative correlation between FA and improvement in motor performance showed evidence for the presence of crossing fibers (Figs. S9, S10, available at www. jneurosci.org as supplemental material). Since FA is a relative value to infer microstructural properties between diffusion directions (Beaulieu, 2002; Mori and Zhang, 2006), we cannot dissociate between diffusivity changes in the parallel or perpendicular diffusion direction from FA alone. Considering recent findings of simultaneous FA and parallel diffusivity changes as a correlate of reading training (Keller and Just, 2009), we tested for learninginduced changes in parallel, perpendicular diffusivity, partial volume fractions, and fiber angle. We interpret our findings as performance-related decrease in FA and diffusivity due to learning-related increase in cell density or axonal/dendritic arborization hindering water diffusion (Jones and Greenough, 1996; Jones et al., 1996; Markham and Greenough, 2004; Kleim et al., 2007).

We also found negative correlation between changes in motor performance and MD in bilateral centrum semiovale (Fig. 4C) indicating a gradually decreasing amount of water diffusion within these regions relative to individual performance improvements across the whole learning period. Notably, performancerelated $\mathrm{MD}$ changes occurred in close spatial proximity to transient, initial gray matter changes in bilateral SMA (Fig. 3A), suggesting a shift of learning-induced structural plasticity from intracortical gray matter to subcortical white matter regions (Armstrong-James et al., 1994).

We now discuss the potential sources that explain the discrepancy between previous findings of learning-dependent FA increases and the FA reduction in our study (Keller and Just, 2009; Scholz et al., 2009; Takeuchi et al., 2010). First, we acknowledge the significant difference in practice intensity and duration between the studies. Keller and Just (2009) reported FA increases after learning periods of 6 months with $\sim 100 \mathrm{~h}$ of practice. Scholz et al. (2009) found FA increases after 6 weeks with five training days per week, and Takeuchi et al. (2010) did so after 2 months of daily practice. Here, we report FA changes (decrease) during 6 weeks of learning with only one training day in each week. Second, from a methodological point of view we introduce in our FA analysis a novel step with adjustment for the hypothesized and detected learning-related volume changes. The question regarding the necessity for intraindividual adjustment of parameter data (e.g., FA, MD, etc.) for the effects of linear/nonlinear interpolation during spatial registration is a fundamental issue that has not been addressed fully in previous studies. As demonstrated in aging-related brain atrophy, there is high correlation between volume changes and FA changes obtained using the VBM approach (Hugenschmidt et al., 2008). We developed a generative approach for tackling this problem and believe that our inferences based on data with preserved initial total signal after linear/ nonlinear interpolation represent more truly the idea of statistical parametric mapping of structural (semi)quantitative data. Third, the direction of FA changes may vary depending on the affected white matter region owing to differences in underlying fiber anatomy (e.g., crossing fibers) relative to task-engaged fiber structures. There could be similar cellular changes in different white matter regions generated by different learning stimuli, however, causing inverse changes in FA based on specificity of task-engaged fibers and their proportion to other fibers within the voxel (Jbabdi et al., 2010). Finally, FA changes and their underlying cellular mechanisms may depend on the extent of taskrelevant neural information processing for complex motor performance either within (local) or between (distributed) brain regions (Karni et al., 1995; Sun et al., 2007). In our study, gradual FA and diffusivity $\left(\lambda_{\|}, \lambda_{\perp}\right.$, and f) (see Fig. S10, available at www. jneurosci.org as supplemental material) decreases in prefrontal white matter regions adjoining cortical areas showing gradual gray matter volume increases may suggest enhanced information processing capacities established through local structural plasticity in neuronal and glia cell components within the apex of the executive system (Markham and Greenough, 2004; Koechlin and Hyafil, 2007).

\section{Limitations of the current study}

Our study is, however, limited by the small number of scanning time points (four scans for each subject) relative to the number of practice sessions ( ear correlation represents the most conservative model with the fewest assumptions regarding temporal dynamics of learningrelated structural changes. The moderate number of four observations (i.e., scanning sessions) in our study does not allow for unambiguous statistical inferences regarding nonlinear effects of structural plasticity.

\section{Conclusion}

Our findings make clear how slight but powerful everyday life experiences could affect adult brain structure. The interrelated structural reorganization in GM and WM points to a causal relationship between behavioral adaptation and structural brain plasticity. Furthermore, the present results extend the recently published findings (Keller and Just, 2009; Scholz et al., 2009; Takeuchi et al., 2010) of white matter FA changes after learning by giving novel and detailed insights into possible underlying neural mechanism and their temporal dynamics during learning.

\section{References}

Adkins DL, Boychuk J, Remple MS, Kleim JA (2006) Motor training induces experience-specific patterns of plasticity across motor cortex and spinal cord. J Appl Physiol 101:1776-1782.

Anderson BJ, Li X, Alcantara AA, Isaacs KR, Black JE, Greenough WT (1994) Glial hypertrophy is associated with synaptogenesis following motor-skill learning, but not with angiogenesis following exercise. Glia 11:73-80.

Andres FG, Mima T, Schulman AE, Dichgans J, Hallett M, Gerloff C (1999) Functional coupling of human cortical sensorimotor areas during bimanual skill acquisition. Brain 122:855-870.

Armstrong-James M, Diamond ME, Ebner FF (1994) An innocuous bias in whisker use in adult rats modifies receptive fields of barrel cortex neurons. J Neurosci 14:6978-6991.

Ashburner J, Friston KJ (2000) Voxel-based morphometry-the methods. Neuroimage 11:805-821

Ashburner J, Friston KJ (2005) Unified segmentation. Neuroimage 26:839851.

Beaulieu C (2002) The basis of anisotropic water diffusion in the nervous system - a technical review. NMR Biomed 15:435-455.

Behrens TE, Berg HJ, Jbabdi S, Rushworth MF, Woolrich MW (2007) Probabilistic diffusion tractography with multiple fibre orientations: what can we gain? Neuroimage 34:144-155.

Boorman ED, Behrens TE, Woolrich MW, Rushworth MF (2009) How green is the grass on the other side? Frontopolar cortex and the evidence in favor of alternative courses of action. Neuron 62:733-743.

Boyke J, Driemeyer J, Gaser C, Büchel C, May A (2008) Training-induced brain structure changes in the elderly. J Neurosci 28:7031-7035.

Butz M, Wörgötter F, van Ooyen A (2009) Activity-dependent structural plasticity. Brain Res Rev 60:287-305.

Caillou N, Delignieres D, Nourrit D, Deschamps T, Lauriot B (2002) Over- 
coming spontaneous patterns of coordination during the acquisition of a complex balancing task. Can J Exp Psychol 56:283-293.

Chklovskii DB, Mel BW, Svoboda K (2004) Cortical rewiring and information storage. Nature 431:782-788.

DeBello WM (2008) Micro-rewiring as a substrate for learning. Trends Neurosci 31:577-584.

Dieterich M, Brandt T (2008) Functional brain imaging of peripheral and central vestibular disorders. Brain 131:2538-2552.

Draganski B, May A (2008) Training-induced structural changes in the adult human brain. Behav Brain Res 192:137-142.

Draganski B, Gaser C, Busch V, Schuierer G, Bogdahn U, May A (2004) Neuroplasticity: changes in grey matter induced by training. Nature 427:311-312.

Driemeyer J, Boyke J, Gaser C, Büchel C, May A (2008) Changes in gray matter induced by learning-revisited. PLoS ONE 3:e2669.

Floyer-Lea A, Matthews PM (2005) Distinguishable brain activation networks for short- and long-term motor skill learning. J Neurophysiol 94:512-518.

Friston KJ, Holmes A, Poline JB, Price CJ, Frith CD (1996) Detecting activations in PET and fMRI: levels of inference and power. Neuroimage 4:223-235.

Guldin WO, Grüsser OJ (1998) Is there a vestibular cortex? Trends Neurosci 21:254-259.

Hayasaka S, Phan KL, Liberzon I, Worsley KJ, Nichols TE (2004) Nonstationary cluster-size inference with random field and permutation methods. Neuroimage 22:676-687.

Hugenschmidt CE, Peiffer AM, Kraft RA, Casanova R, Deibler AR, Burdette JH, Maldjian JA, Laurienti PJ (2008) Relating imaging indices of white matter integrity and volume in healthy older adults. Cereb Cortex 18:433-442.

Jbabdi S, Behrens TE, Smith SM (2010) Crossing fibres in tract-based spatial statistics. Neuroimage 49:249-256.

Jones TA, Greenough WT (1996) Ultrastructural evidence for increased contact between astrocytes and synapses in rats reared in a complex environment. Neurobiol Learn Mem 65:48-56.

Jones TA, Hawrylak N, Greenough WT (1996) Rapid laminar-dependent changes in GFAP immunoreactive astrocytes in the visual cortex of rats reared in a complex environment. Psychoneuroendocrinology 21:189201.

Kable JW, Glimcher PW (2009) The neurobiology of decision: consensus and controversy. Neuron 63:733-745.

Karni A, Meyer G, Jezzard P, Adams MM, Turner R, Ungerleider LG (1995) Functional MRI evidence for adult motor cortex plasticity during motor skill learning. Nature 377:155-158.

Karni A, Meyer G, Rey-Hipolito C, Jezzard P, Adams MM, Turner R, Ungerleider LG (1998) The acquisition of skilled motor performance: fast and slow experience-driven changes in primary motor cortex. Proc Natl Acad Sci U S A 95:861-868.

Keller TA, Just MA (2009) Altering cortical connectivity: remediationinduced changes in the white matter of poor readers. Neuron 64:624-631.

Kleim JA, Markham JA, Vij K, Freese JL, Ballard DH, Greenough WT (2007) Motor learning induces astrocytic hypertrophy in the cerebellar cortex. Behav Brain Res 178:244-249.

Koechlin E, Hyafil A (2007) Anterior prefrontal function and the limits of human decision-making. Science 318:594-598.

Koechlin E, Basso G, Pietrini P, Panzer S, Grafman J (1999) The role of the anterior prefrontal cortex in human cognition. Nature 399:148-151.

Langhorne P, Coupar F, Pollock A (2009) Motor recovery after stroke: a systematic review. Lancet Neurol 8:741-754.

Lee JE, Chung MK, Lazar M, DuBray MB, Kim J, Bigler ED, Lainhart JE, Alexander AL (2009) A study of diffusion tensor imaging by tissue- specific, smoothing-compensated voxel-based analysis. Neuroimage 44:870-883.

Lee TD, Swinnen SP (1993) Three legacies of Bryan and Harter: Automaticity, variability and change in skilled performance. In: Cognitive issues in motor expertise (Starkes JL, Allard F, eds), pp 27-56. Amsterdam: North-Holland.

Luft AR, Buitrago MM (2005) Stages of motor skill learning. Mol Neurobiol 32:205-216.

Markham JA, Greenough WT (2004) Experience-driven brain plasticity: beyond the synapse. Neuron Glia Biol 1:351-363.

May A, Gaser C (2006) Magnetic resonance-based morphometry: a window into structural plasticity of the brain. Curr Opin Neurol 19:407-411.

May A, Hajak G, Gänssbauer S, Steffens T, Langguth B, Kleinjung T, Eichhammer P (2007) Structural brain alterations following 5 days of intervention: dynamic aspects of neuroplasticity. Cereb Cortex 17:205210.

Mori S, Zhang J (2006) Principles of diffusion tensor imaging and its applications to basic neuroscience research. Neuron 51:527-539.

Moulton CA, Dubrowski A, Macrae H, Graham B, Grober E, Reznick R (2006) Teaching surgical skills: what kind of practice makes perfect?: a randomized, controlled trial. Ann Surg 244:400-409.

Nachev P, Kennard C, Husain M (2008) Functional role of the supplementary and pre-supplementary motor areas. Nat Rev Neurosci 9:856-869.

Newell KM (1996) Change in movement and skill: learning, retention and transfer. In: Dexterity and its development (Latash ML, Turvey MT, eds). Mahwah, NJ: Erlbaum.

Newell KM, Kugler PN, van Emmerik REA, MacDonald PV (1989) Search strategies and the acquisition of coordination. In: Perspectives on the coordination of movement (Wallace SA, ed), pp 85-122. Amsterdam: North-Holland.

Oldfield RC (1971) The assessment and analysis of handedness: the Edinburgh inventory. Neuropsychologia 9:97-113.

Orrell AJ, Eves FF, Masters RS (2006) Implicit motor learning of a balancing task. Gait Posture 23:9-16.

Passingham R (1993) The Frontal Lobes and Voluntary Action. Oxford: Oxford UP.

Ryan ED (1965) Retention of stabilometer performance over extended periods of time. Res Q 36:46-51.

Schmidt RA, Lee TD (1999) Motor control and learning: a behavioral emphasis. Champaign, IL: Human Kinetics.

Scholz J, Klein MC, Behrens TE, Johansen-Berg H (2009) Training induces changes in white-matter architecture. Nat Neurosci 12:1370-1371.

Sun FT, Miller LM, Rao AA, D’Esposito M (2007) Functional connectivity of cortical networks involved in bimanual motor sequence learning. Cereb Cortex 17:1227-1234.

Swinnen SP, Wenderoth N (2004) Two hands, one brain: cognitive neuroscience of bimanual skill. Trends Cogn Sci 8:18-25.

Takeuchi H, Sekiguchi A, Taki Y, Yokoyama S, Yomogida Y, Komuro N, Yamanouchi T, Suzuki S, Kawashima R (2010) Training of working memory impacts structural connectivity. J Neurosci 30:3297-3303.

Trachtenberg JT, Chen BE, Knott GW, Feng G, Sanes JR, Welker E, Svoboda $\mathrm{K}$ (2002) Long-term in vivo imaging of experience-dependent synaptic plasticity in adult cortex. Nature 420:788-794.

Wulf G, Weigelt M, Poulter D, McNevin N (2003) Attentional focus on suprapostural tasks affects balance learning. Q J Exp Psychol A 56:1191-1211.

Xu T, Yu X, Perlik AJ, Tobin WF, Zweig JA, Tennant K, Jones T, Zuo Y (2009) Rapid formation and selective stabilization of synapses for enduring motor memories. Nature 462:915-919.

Zanone PG, Kelso JA (1992) Evolution of behavioral attractors with learning: nonequilibrium phase transitions. J Exp Psychol Hum Percept Perform 18:403-421. 